\title{
Maternal traditional dietary pattern and antiretroviral treatment exposure are associated with neonatal size and adiposity in urban, black South Africans
}

\author{
Stephanie V. Wrottesley ${ }^{1 *}$, Ken K. Ong ${ }^{1,2}$, Pedro T. Pisa ${ }^{1,3}$ and Shane A. Norris ${ }^{1}$ \\ ${ }^{1}$ Medical Research Council/Wits Developmental Pathways for Health Research Unit, Department of Paediatrics, \\ Faculty of Health Sciences, University of the Witwatersrand, Johannesburg, 1862, South Africa \\ ${ }^{2}$ Medical Research Council Epidemiology Unit, University of Cambridge, Cambridge CB2 OSL, UK \\ ${ }^{3}$ Wits Reproductive Health and HIV Institute, University of the Witwatersrand, Johannesburg, 2001, South Africa \\ (Submitted 20 December 2017 - Final revision received 28 May 2018 - Accepted 30 May 2018 - First published online 30 July 2018 )
}

\section{Abstract}

This study examines the associations between maternal Traditional dietary pattern adherence and HIV/treatment with neonatal size and adiposity in urban, black South Africans, as well as how specific maternal factors - that is BMI and gestational weight gain (GWG) - may influence these associations. Multiple linear regression models were used to examine associations among maternal Traditional diet pattern adherence (pattern score), HIV/treatment status (three groups: HIV negative, HIV positive (antenatal antiretroviral treatment (ART) initiation), HIV positive (pre-pregnancy ART initiation)), BMI and GWG (kg/week), and newborn (1) weight:length ratio (WLR, kg/m) in 393 mother-neonate pairs, and (2) Peapod estimated fat mass index $\left(\mathrm{FMI}, \mathrm{kg} / \mathrm{m}^{3}\right)$ in a 171-pair subsample. In fully adjusted models, maternal obesity and GWG were associated with $0.25 \mathrm{~kg} / \mathrm{m}(P=0.008)$ and $0.48 \mathrm{~kg} / \mathrm{m}(P=0.002)$ higher newborn WLR, whereas Traditional diet pattern score was associated with lower newborn WLR $(-0.04 \mathrm{~kg} / \mathrm{m}$ per $+1 \mathrm{sD} ; P=0.033)$. In addition, Traditional diet pattern score was associated with $0 \cdot 13 \mathrm{~kg} / \mathrm{m}^{3}(P=0 \cdot 027)$ and $0.32 \mathrm{~kg} / \mathrm{m}^{3}(P=0.005)$ lower FMI in the total sample and in newborns of normal-weight women, respectively. HIV-positive (pre-pregnancy ART) $v$. HIV-negative (ref) status was associated with $1.11 \mathrm{~kg} / \mathrm{m}^{3}(P=0.002)$ higher newborn FMI. Promotion of a Traditional dietary pattern, alongside a healthy maternal pre-conception weight, in South African women may reduce newborn adiposity and metabolic risk profiles. In HIV-positive women, targeted monitoring and management strategies are necessary to limit treatment-associated effects on in utero fat deposition.

\section{Key words: Pregnancy: Traditional dietary patterns: Antiretroviral treatment: Neonatal adiposity: South Africa}

Maternal pre-pregnancy BMI and gestational weight gain (GWG) are established predictors of fetal growth and birth outcomes, with both obesity and excessive weight gain being associated with high birth weight and large-for-gestational age deliveries, as well as with obesity and cardiometabolic disease risk in later life ${ }^{(1-5)}$. Although the effects of anthropometrically defined maternal nutritional status on birth weight have been well documented, the influence of dietary patterns on birth size - particularly in increasingly urbanised low- and middleincome countries such as South Africa - is not known. In addition, the predominant use of birth weight as a proxy for fetal growth fails to elucidate the effects of diet on adiposity (i.e. fat $v$. fat-free mass) and therefore provides only a weak indication of newborn metabolic risk ${ }^{(6,7)}$. Studies have shown that adiposity in early infancy tends to track through childhood and is associated with a long-term risk of central adiposity, as well as of elevated TAG levels and insulin resistance ${ }^{(8,9)}$. Finally, the extent to which high HIV and antiretroviral treatment (ART) exposure in this setting before, and during, pregnancy may affect these associations is yet to be explored.

During a previous study in urban, black South Africans, we used principal component analysis (PCA) to identify three distinct dietary patterns in pregnant women, namely Western, Traditional and Mixed ${ }^{(10)}$. These patterns were consistent with those expected for a transitioning African population experiencing a shift towards increasingly westernised diets high in saturated fat, sugar, salt, processed/convenience foods and edible oils and low in essential micronutrients ${ }^{(11,12)}$. We further showed that adherence to the Traditional dietary pattern characterised by high intakes of vegetables, beans and legumes, traditional meats and whole grains - was associated with a lower rate of GWG and reduced odds of excessive weight gain. These associations remained evident in women of normal weight after stratification, but not in overweight or obese subgroups. This suggests that the relationship between diet and maternal adiposity may be modified by baseline BMI. However,

Abbreviations: ADP, air displacement plethysmography; ART, antiretroviral treatment; DXA, dual-energy X-ray absorptiometry; FMI, fat mass index; GWG, gestational weight gain; QFFQ, quantitative FFQ; WLR, weight:length ratio.

* Corresponding author: S. V. Wrottesley, email stephanie.wrottesley@gmail.com 
whether Traditional pattern intake is similarly associated with beneficial reductions in neonatal adiposity - either independently or via interactions with maternal nutritional status - is not known.

The maternal high-risk profile in this setting - where $66 \%$ of women are overweight or obese and $55 \%$ experience excessive GWG - is further complicated by a $33 \%$ prevalence of $\mathrm{HIV}^{(10)}$. Although ART initiation is mandatory in South Africa for all HIVpositive pregnant women not yet receiving treatment, and this has had undeniable benefits in the prevention of vertical transmission, the metabolic consequences for both mother and infant are not clear. Both HIV infection and ART exposure have been positively associated with weight and fat distribution changes and altered glucose and lipid metabolism in both adults and children, as well as with adverse birth outcomes $^{(13-17)}$. However, little is known about the possible effects of HIV/treatment on fetal growth and adiposity, as well the risk of non-communicable disease in the long term.

The aim of this study was therefore to examine the associations between maternal Traditional dietary pattern adherence and HIV/treatment with neonatal size and adiposity in urban, black South Africans, as well as how specific maternal factors that is BMI and GWG - may influence these associations.

\section{Methods \\ Study setting and participants}

This study was nested within a large pregnancy cohort study (Soweto First 1000-Day Study; S 1000), based at the Chris Hani Baragwanath Hospital in Soweto, Johannesburg, South Africa, between 2013 and 2016. Overall, S 1000 aimed to understand the complex associations between multiple maternal factors and fetal and infant outcomes in an urban-poor African context, and to identify the levers that could optimise maternal and child health within the first $1000 \mathrm{~d}$. Inclusion criteria for S 1000 were as follows: resident of Soweto, or the Greater Soweto area, $<20$ weeks gestational age at recruitment, non-epileptic, nondiabetic, 18 years or older and pregnant with a singleton, naturally conceived pregnancy. Data collection for S 1000 took place at six time points during pregnancy ( $<14$ weeks; $14-$ 18 weeks; 19-23 weeks; 24-28 weeks; 29-33 weeks; and 3438 weeks) and eight time points after delivery ( $<14 \mathrm{~d}$; 6 weeks; 2 months; 3 months; 6 months; 12 months; 18 months; and 24 months). All women provided written informed consent before their inclusion in the pregnancy component of the study (Soweto Fetal Growth Study), as well as before the inclusion of themselves and their infants in the post-delivery follow-up (Soweto Baby Growth Study). Ethical approval was obtained from the University of the Witwatersrand's Research Ethics Committee (Medical) for both components of S 1000 (M120524 and M130905). In all, 559 women were recruited into this substudy and had dietary intake assessed at 14-18 weeks.

\section{Maternal variables}

Demographic, health and socio-economic variables. Maternal demographic and socio-economic variables were collected by trained members of research staff using intervieweradministered questionnaires at the first pregnancy visit ( $<14$ weeks gestational age). Parity was defined as the number of previous births at a gestational age of 24 weeks or more, regardless of whether the infant was born alive or was stillborn. Smoking and/or chewing tobacco was reported at baseline. HIV status was self-reported at each pregnancy visit and confirmed using the results recorded in the participant's antenatal clinic card. According to South Africa's national Prevention of Motherto-Child Transmission guidelines, routine HIV counselling and testing is required during pregnancy; for any HIV-positive woman who is not already receiving treatment, ART is initiated. All HIV-positive participants in this study were therefore receiving ART and were stratified according to whether they had been initiated on ART before pregnancy (pre-pregnancy ART) or during the current pregnancy (antenatal ART). Household socio-economic status (SES) was assessed using an asset index, which scored each participant according to the number of assets that they possessed out of a possible nine (electricity, radio, television, refrigerator, mobile phone, personal computer, bicycle, motorcycle/scooter, car). This was based on standard measures used in the Demographic and Health Surveys household questionnaire (available at: www.measuredhs.com) and has been extensively used in this setting ${ }^{(18,19)}$. Asset index scores were subsequently grouped into low $(<5)$, medium $(5-7)$ and high (>7) SES categories. Maternal education was defined according to the highest level of education completed (primary, secondary or tertiary).

Anthropometry. A wall-mounted Stadiometer (Holtain) was used to measure maternal height to the nearest $1 \mathrm{~mm}$ at baseline. Maternal weight was measured to the nearest $0.1 \mathrm{~kg}$ at each visit during pregnancy using a digital scale. Weight at recruitment ( $<14$ weeks) was used as a proxy for prepregnancy weight and, together with height, was used to calculate maternal BMI (weight $(\mathrm{kg}) /$ height $\left(\mathrm{m}^{2}\right)$ ). There were no underweight women in this sample, and therefore BMI was classified according to the following categories: normal weight $\left(18.5-24.9 \mathrm{~kg} / \mathrm{m}^{2}\right)$, overweight $\left(25-29.9 \mathrm{~kg} / \mathrm{m}^{2}\right)$ or obese $\left(\geq 30.0 \mathrm{~kg} / \mathrm{m}^{2}\right)$. GWG ( $\mathrm{kg} /$ week) was calculated as follows: ((weight at final pregnancy visit - weight at recruitment)/duration of follow-up). GWG was classified as inadequate, adequate or excessive according to the BMI-specific weight-gain ranges recommended by the Institute of Medicine ${ }^{(20)}$.

Dietary intake. Habitual dietary intake was assessed at the second pregnancy visit (14-18 weeks) using an intervieweradministered quantitative FFQ (QFFQ). This nationally used QFFQ was developed by the South African Medical Research Council based on analyses of eleven dietary surveys conducted in rural and urban South Africa, and includes all foods consumed by at least $3 \%$ of the population ${ }^{(21)}$. Retrospective data were collected on the frequency and quantity of food and beverage intake during the previous week using food flash cards (high-quality photographs of food items) and a combination of household measures, two-dimensional life-size drawings of foods and utensils and three-dimensional food 
models as described and validated by Steyn et al. ${ }^{(22)}$. According to the criteria developed by Dennis et al., this QFFQ is a very high-quality tool - scoring a total of thirteen points (high quality classified as a score of seven or higher) ${ }^{(23)}$. This QFFQ has been extensively piloted and used in this setting, and results are published elsewhere ${ }^{(10,24,25)}$. QFFQ data were captured electronically using REDCap electronic data capture tools hosted at The University of the Witwatersrand ${ }^{(26)}$.

Neonatal variables. Neonates were included in this study if they were born at term ( $\geq 37$ weeks) and had complete delivery outcome data. In addition, body composition was analysed for a sub-sample that had assessments via either air displacement plethysmography (ADP) using the Peapod (Cosmed) or dualenergy X-ray absorptiometry (DXA; Hologic DiscoveryA S/N 86254, APEX software version 4.0.2; Hologic Inc.) within the first 2 weeks of life.

Birth outcomes. Gestational age at delivery (weeks) was calculated as follows: (duration of pregnancy follow-up (date of delivery-date of baseline ultrasound dating scan) + gestational age at baseline (crown-to-rump length measured by ultrasound; d)). Birth weight and length were measured by trained research nurses within $24 \mathrm{~h}$ of delivery for $82 \%$ of neonates. Where assessment within this window was not possible - for example because of the infant being admitted to the hospital for observation - measurements were taken within $48 \mathrm{~h}$. Weight: length ratio (WLR; $\mathrm{kg} / \mathrm{m}$ ) was calculated to represent the best anthropometric predictor of neonatal body composition (fat-free mass and fat mass) as described by Villar et al. ${ }^{(27)}$.

Neonatal body composition. Weight and length were measured and fat mass and fat-free mass estimated for nude neonates within $14 \mathrm{~d}$ of birth. According to a previous study in which we demonstrated the level of agreement between ADP and DXA estimated body composition in this population, ADP was used where available ${ }^{(28)}$. In cases in which a neonate had only DXA measurements, fat mass and fat-free mass were converted to their ADP equivalent estimates using the following linear equations:

$$
\text { Fat mass (ADP equivalent })=139 \cdot 8311+0 \cdot 7974718
$$$$
\times \text { DXA fat mass: }
$$

Fat-free mass $($ ADP equivalent $)=89 \cdot 40371+0.8728309$

$\times$ DXA fat-free mass.

These equations were generated for the population from the regression of $\mathrm{ADP}$ on DXA measurements in the aforementioned ADP/DXA comparison study and provided reliable ADP equivalent estimates $^{(28)}$. Fat mass index (FMI; $\mathrm{kg} / \mathrm{m}^{3}$ ) was calculated from these estimates to describe adiposity in neonates. As described by Villar et al., the applicability of exponents in body composition indices to address the relationship between body composition and size may vary across populations ${ }^{(27)}$. We therefore regressed fat mass on length (data in natural logarithms) to confirm that this index $\left(\mathrm{kg} / \mathrm{m}^{3}\right)$ provided the best description of the relationship between weight and length in the study population. The applicability of the power exponent was confirmed; regression power exponent: $2 \cdot 8$ (SE 0.6) ${ }^{(29)}$.

Air displacement plethysmography. Peapod assessments were performed according to standard procedures as previously described ${ }^{(30,31)}$. Participants were placed inside the Peapod chamber wearing only a wig cap if necessary. Body volume was estimated using pressure and volume changes (air displacement) within the chamber and body density calculated using body mass and volume measurements. Fat mass and fatfree mass were subsequently derived using sex-specific equations developed by Fomon et al. ${ }^{(32)}$

Dual-energy $X$-ray absorptiometry. DXA scanning was performed according to standard procedures as described elsewhere $^{(33)}$. Typically, neonates were fed before DXA scanning and were sleeping during the procedure. Neonates were placed supine on the scanning bed wearing only a disposable diaper and swaddled in a cotton blanket. Scans were satisfactory for use if the subject's body lay within the scanning region and there was minimal movement during assessment. Whole-body measurements of fat mass and fat-free mass were extracted for use in analyses.

\section{Statistical analysis}

Data were analysed for 393 mother-neonate pairs with complete data using STATA 13.0 (StataCorp). The flow of participants through the sub-study to reach the final sample sizes for the primary and secondary outcomes (WLR and FMI, respectively) is depicted in online Supplementary Fig. S1. Mother-neonate pairs included in the final analyses did not differ in any baseline maternal characteristics (demographics, SES and anthropometry) from those excluded.

The dietary patterns previously identified in this population namely Western, Traditional and Mixed - were confirmed in this sub-sample using PCA, as described elsewhere ${ }^{(10)}$. PCA was conducted using orthogonal (varimax) rotation on the weekly frequency of consumption of the QFFQ food items, classified as forty-eight food items/groups based on those described by Crozier et al. ${ }^{(34,35)}$. The Kaiser-Meyer-Olkin measure of sampling adequacy $(0.68)$ and Bartlett's test of sphericity $(P<0.001)$ confirmed PCA as an appropriate dimension reduction technique for use in this sample. Eigen values, as well as their visual inflections on a scree plot, and the percentage of total variance explained were used to retain patterns. As described elsewhere, foods or food groups with factor loadings $\geq 0 \cdot 2$ reflected strong associations with principal components and were used to name the dietary patterns ${ }^{(36)}$. Dietary pattern scores for each pattern were generated by multiplying factor loadings by the standardised intake of each food/food group and then summing these. Mean factor scores for the patterns were zero, with positive and negative scores representing high and low adherence, respectively, of each dietary pattern ${ }^{(37)}$. Because of the associations previously demonstrated between the Traditional diet pattern and GWG in this population, maternal diet was classified according to adherence to the Traditional pattern (Traditional pattern score) in all subsequent study analyses. 
Maternal and neonatal characteristics of the sample are presented as medians and interquartile ranges and percentages and numbers for continuous and categorical variables, respectively. The Kruskal-Wallis test was used to compare neonatal WLR and FMI according to the following maternal and infant factors: maternal age, parity, HIV/treatment status, smoking status, education, marital status, SES, BMI at recruitment, GWG, Traditional diet pattern adherence, neonate sex and gestational age at birth.

On the basis of known associations between maternal factors and birth size, as well as previously described associations in this population ${ }^{(10)}$, we proposed a conceptual framework for the associations between maternal Traditional pattern adherence (continuous: diet pattern score), BMI (categorical: normal weight (ref) $v$. overweight and obese), GWG (continuous: $\mathrm{kg} /$ week), HIV/treatment status (categorical: HIV negative (ref) $v$. HIV positive (antenatal ART) and HIV positive (pre-pregnancy ART)) and neonatal WLR (continuous: $\mathrm{kg} / \mathrm{m}$ ) and FMI (continuous: $\mathrm{kg} / \mathrm{m}^{3}$ ). The bivariate associations between these maternal factors and each neonatal outcome were tested using linear regression analyses.

To identify the independent associations between diet, BMI, GWG, HIV/treatment status and WLR and FMI, we performed hierarchical regression analyses per outcome. Covariates included in analyses were maternal or infant variables conclusively associated with infant outcome(s) (namely parity, newborn sex and gestational age at birth). In addition, for FMI, age at scan (d) was included to adjust for variation across the 2-week assessment period. Regression coefficients and $R^{2}$ values are therefore presented across three models for WLR and FMI. Variables included in the models were as follows: model 1 (M1), neonate sex and Traditional diet pattern score; model 2 (M2), M1 with HIV/treatment status, BMI and GWG; and model 3 (M3), M2 with parity and gestational age at delivery (and age at scan for FMI). To test for an interaction between BMI and dietary pattern adherence, a fourth model per outcome was run as follows: M3 with the interaction term BMI category $\times$ Traditional pattern score (data not shown). $P$ values tailed at 0.05 were considered statistically significant.

\section{Results}

Maternal and infant characteristics are presented in Table 1. The median age of pregnant women was 30 years. In all, 35 and $30 \%$ of women were overweight and obese at recruitment, respectively, whereas $58 \%$ gained excessive weight according to the IoM BMI-specific guidelines; $34 \%$ of women were HIV positive, with $23 \%$ in total being initiated on ART during the current pregnancy. A total of $52 \%$ of newborns were male. Neonates had a median birth weight of $3100 \mathrm{~g}$ and WLR and FMI were $6.4 \mathrm{~kg} / \mathrm{m}$ and $3.6 \mathrm{~kg} / \mathrm{m}^{3}$, respectively.

As previously described, for the purpose of this study maternal diet was classified according to Traditional diet pattern adherence (i.e. Traditional diet pattern score). This dietary pattern was characterised by high factor loadings for vegetables, beans and legumes, traditional meats and porridge/pap (online Supplementary Table S1).
Table 1. Maternal and neonatal characteristics of urban, black South Africans ( $n$ 393)

(Medians and interquartile ranges (IQR); percentages)

\begin{tabular}{|c|c|c|}
\hline & Median & IQR \\
\hline \multicolumn{3}{|l|}{ Maternal variables } \\
\hline \multicolumn{3}{|l|}{ Demographic and health characteristics } \\
\hline Maternal age (years) & 30 & $25-35$ \\
\hline \multicolumn{3}{|l|}{ Parity } \\
\hline Parity 0 & \multicolumn{2}{|r|}{25} \\
\hline Parity 1 & \multicolumn{2}{|r|}{45} \\
\hline Parity $\geq 2$ & \multicolumn{2}{|r|}{30} \\
\hline \multicolumn{3}{|l|}{ HIV status } \\
\hline HIV-negative & \multicolumn{2}{|r|}{66} \\
\hline HIV-positive (antenatal ART) & \multicolumn{2}{|r|}{23} \\
\hline HIV-positive (pre-pregnancy ART) & \multicolumn{2}{|r|}{11} \\
\hline \multicolumn{3}{|l|}{ Smokes/chews tobacco } \\
\hline No & \multirow{2}{*}{\multicolumn{2}{|c|}{$\begin{array}{l}87 \\
13\end{array}$}} \\
\hline Yes & & \\
\hline \multicolumn{3}{|l|}{ Socio-economic characteristics } \\
\hline \multicolumn{3}{|l|}{ Maternal education } \\
\hline Primary & \multicolumn{2}{|r|}{2} \\
\hline Secondary & \multicolumn{2}{|r|}{70} \\
\hline Tertiary & \multicolumn{2}{|r|}{28} \\
\hline \multicolumn{3}{|l|}{ Marital status ( $n$ 387) } \\
\hline Single & \multirow{2}{*}{\multicolumn{2}{|c|}{$\begin{array}{l}61 \\
39\end{array}$}} \\
\hline Married/cohabiting & & \\
\hline \multicolumn{3}{|l|}{ Household SES } \\
\hline Low & \multicolumn{2}{|r|}{13} \\
\hline Medium & \multicolumn{2}{|r|}{80} \\
\hline High & \multicolumn{2}{|r|}{7} \\
\hline Anthropometry & & \\
\hline BMI at recruitment $\left(\mathrm{kg} / \mathrm{m}^{2}\right)(<14$ weeks) & $27 \cdot 2$ & $23.8-30.9$ \\
\hline Normal weight (18.5-24.9) & & 35 \\
\hline Overweight (25-29.9) & & 35 \\
\hline Obese $(\geq 30)$ & & 30 \\
\hline GWG (kg/week) & 0.41 & $0.28-0.56$ \\
\hline Inadequate & & 22 \\
\hline Adequate & & 20 \\
\hline Excessive & & 58 \\
\hline Neonatal variables & & \\
\hline Sex & & \\
\hline Male & & 52 \\
\hline Female & & 48 \\
\hline Gestational age at delivery (weeks) & 39 & $38-40$ \\
\hline Anthropometry & & \\
\hline Birth weight $(\mathrm{g})$ & 3100 & $2850-3365$ \\
\hline Birth length $(\mathrm{cm})$ & 48.9 & $47 \cdot 3-50 \cdot 3$ \\
\hline Weight:length ratio $(\mathrm{kg} / \mathrm{m})$ & $6 \cdot 4$ & $5 \cdot 9-6 \cdot 8$ \\
\hline Body composition $(n 171)^{\star}$ & & \\
\hline Age at scanning $(d)$ & 8 & 4 \\
\hline Fat mass $(\mathrm{g})$ & 435 & $331-548$ \\
\hline Fat-free mass $(\mathrm{g})$ & 2774 & $2530-2929$ \\
\hline Fat mass index $\left(\mathrm{kg} / \mathrm{m}^{3}\right)$ & 3.6 & $2 \cdot 9-4.6$ \\
\hline
\end{tabular}

ART, antiretroviral treatment loM GWG ranges ( $\mathrm{kg} /$ week): inadequate, normal weight $<0.35$, overweight $<0.23$, obese $<0.17$; adequate, normal weight $0.35-0.50$, overweight $0.23-0.33$, obese $0.17-0.27$; excessive, normal weight $>0.50$, overweight $>0.33$, obese $>0.27$; GWG, gestational weight gain.

* Measured by air displacement plethysmography (air displacement plethysmography; Peapod) or dual-energy X-ray absorptiometry corrected for the measurement differences between techniques.

Of the maternal variables described as potential covariates in this population, only parity was consistently associated with neonatal outcomes, with higher WLR and FMI seen in infants born to mothers who had experienced at least one previous birth (online Supplementary Table 2). Female neonates had significantly higher FMI than males, and WLR increased with gestational age.

Conceptual models for the bivariate associations between maternal Traditional diet pattern, BMI, GWG and HIV/treatment 


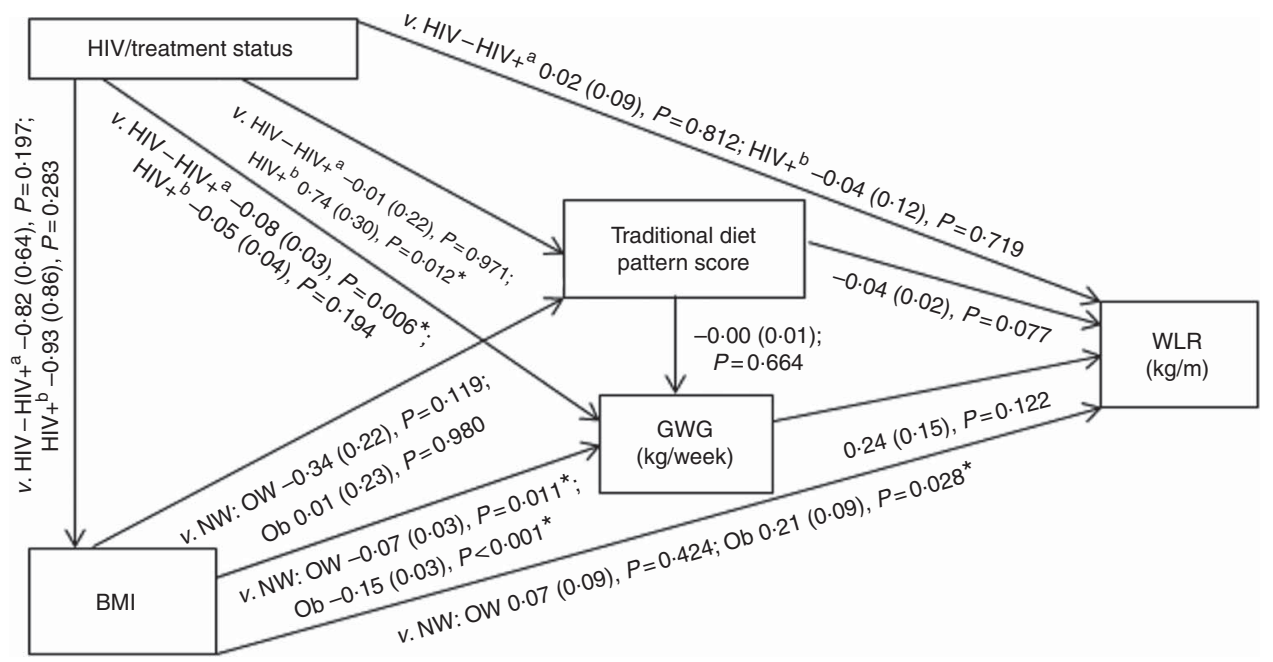

Fig. 1. Conceptual model with bivariate associations between maternal factors and neonatal weight:length ratio $(\mathrm{kg} / \mathrm{m})$ in urban, black South Africans. Values are regression coefficients $(\beta)$ with their standard errors from linear regression analyses. GWG, gestational weight gain; HIV-, HIV negative; HIV ${ }^{a}$, HIV positive (antenatal ART); HIV $+{ }^{\mathrm{b}}$, HIV positive (pre-pregnancy ART); NW, normal weight; OW, overweight; Ob, obese; $v$., compared with the following reference category; WLR, newborn weight:length ratio. ${ }^{*}$ Significant results $(P<0.05)$.

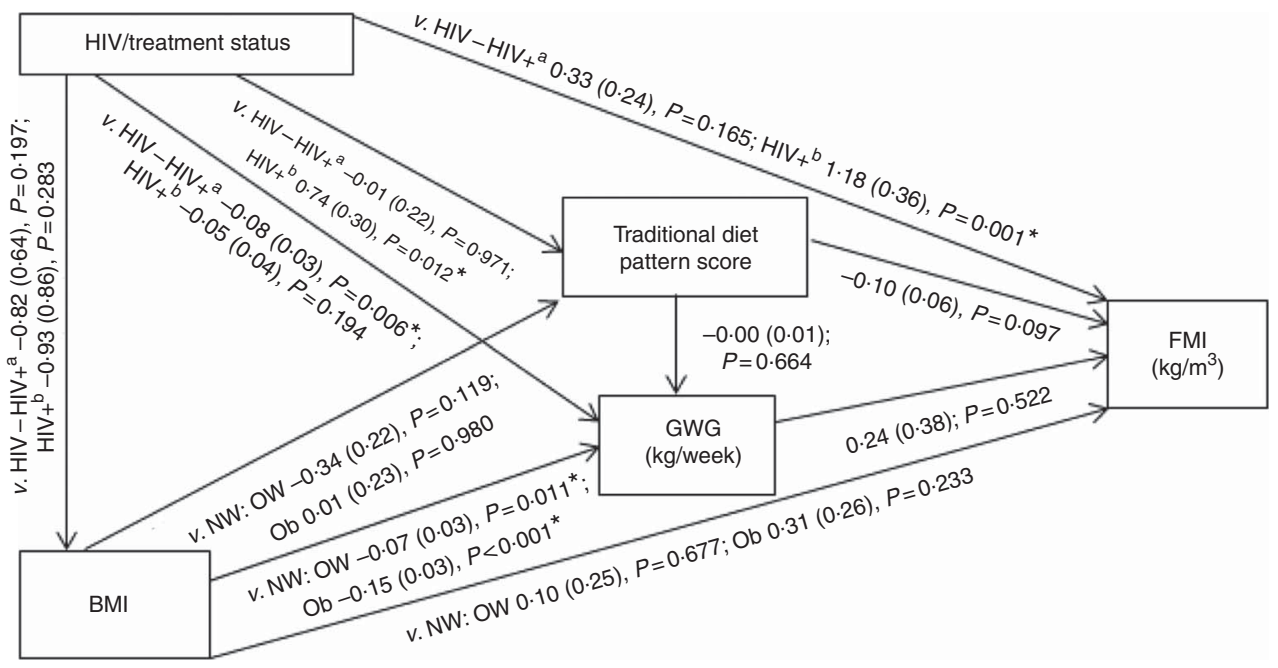

Fig. 2. Conceptual model with bivariate associations between maternal factors and neonatal fat mass index $\left(\mathrm{kg} / \mathrm{m}^{3}\right)$ in urban, black South Africans. Values are regression coefficients $(\beta)$ with standard errors from linear regression analyses. FMI, neonatal fat mass index; GWG, gestational weight gain; HIV-, HIV negative; $\mathrm{HIV}_{+}{ }^{\mathrm{a}}$, HIV positive (antenatal ART); HIV ${ }^{\mathrm{b}}$, HIV positive (pre-pregnancy ART); NW, normal weight; OW, overweight; Ob, obese; $v$., compared with the following reference category. ${ }^{*}$ Significant results $(P<0.05)$.

status and outcomes of interest are presented in Fig. 1 (WLR) and 2 (FMI). Compared with women with normal weight at recruitment, overweight and obese women exhibited significantly lower GWG. GWG was also lower in HIV-positive (antenatal ART initiation) $v$. HIV-negative women. In addition, in HIV-positive women, pre-pregnancy ART initiation was associated with higher adherence to the Traditional diet pattern. Maternal obese $v$. normal-weight BMI was positively associated with WLR during bivariate analyses, whereas HIV-positive (prepregnancy ART) status was positively associated with FMI when compared with HIV-negative status.

Table 2 presents the results of hierarchical regression analyses of maternal variables on newborn WLR. In fully adjusted models (M3: adjusted for neonate sex, Traditional diet pattern score, HIV/treatment status, BMI, GWG, parity and gestational age at delivery) a 1-SD increase in Traditional diet pattern score was inversely associated with newborn WLR $(-0.04 \mathrm{~kg} / \mathrm{m}$; $P=0.033$ ). In addition, compared with a normal-weight BMI at recruitment, maternal obesity was positively associated with WLR (M3: $0.25 \mathrm{~kg} / \mathrm{m} ; P=0.008$ ) and a $1-\mathrm{kg} /$ week increase in GWG was associated with a $0 \cdot 48 \mathrm{-kg} / \mathrm{m}$ increase in newborn WLR (M3: $P=0 \cdot 002)$. M3 explained approximately $14 \%$ of the variation in newborn WLR.

The results of hierarchical regression analyses of maternal variables on newborn FMI are presented in Table 3. Traditional diet pattern adherence was associated with lower FMI $\left(-0.13 \mathrm{~kg} / \mathrm{m}^{3}\right.$ per $\left.+1 \mathrm{sD} ; \quad P=0.027\right)$ after full adjustment for covariates (M3: adjusted for neonate sex, Traditional diet 
Table 2. Hierarchical regression for the associations between maternal factors and neonatal weight:length ratio $(n 393)$ (Regression coefficients $(\beta)$ and $95 \%$ confidence intervals)

\begin{tabular}{|c|c|c|c|c|c|c|c|c|c|}
\hline \multirow[b]{3}{*}{ Independent variables } & \multicolumn{9}{|c|}{ Weight:length ratio $(\mathrm{kg} / \mathrm{m})$} \\
\hline & \multicolumn{3}{|c|}{ Model 1} & \multicolumn{3}{|c|}{ Model 2} & \multicolumn{3}{|c|}{ Model 3} \\
\hline & $\beta$ & $95 \% \mathrm{Cl}$ & $P \dagger$ & $\beta$ & $95 \% \mathrm{Cl}$ & $P \dagger$ & $\beta$ & $95 \% \mathrm{Cl}$ & $P \dagger$ \\
\hline \multicolumn{10}{|l|}{ Neonate sex } \\
\hline Male & & Ref. & & & Ref. & & & Ref. & \\
\hline Female & -0.02 & $-0.17,0.13$ & 0.769 & -0.05 & $-0.19,0.10$ & 0.533 & -0.06 & $-0.20,0.08$ & 0.396 \\
\hline \multicolumn{10}{|l|}{ Maternal dietary pattern } \\
\hline Traditional pattern score & -0.04 & $-0.08,0.00$ & 0.078 & -0.04 & $-0.08,0.00$ & 0.081 & -0.04 & $-0.08,-0.00$ & $0.033^{*}$ \\
\hline \multicolumn{10}{|l|}{ HIV/treatment status } \\
\hline HIV-negative & & & & & Ref. & & & Ref. & \\
\hline HIV-positive (antenatal ART) & & & & 0.07 & $-0.11,0.25$ & 0.422 & 0.07 & $-0.10,0.24$ & 0.436 \\
\hline HIV-positive (pre-pregnancy ART) & & & & 0.01 & $-0.23,0.26$ & 0.906 & -0.02 & $-0.26,0.21$ & 0.833 \\
\hline \multicolumn{10}{|l|}{ BMI category } \\
\hline Normal weight & & & & & Ref. & & & Ref. & \\
\hline Overweight & & & & 0.09 & $-0.09,0.26$ & 0.343 & 0.09 & $-0.08,0.26$ & 0.301 \\
\hline Obese & & & & 0.27 & $0.08,0.46$ & $0.005^{\star}$ & 0.25 & $0.07,0.43$ & $0.008^{*}$ \\
\hline \multicolumn{10}{|l|}{ Gestational weight gain } \\
\hline Rate (kg/week) & & & & 0.36 & $0.05,0.67$ & $0.024^{*}$ & 0.48 & $0.18,0.78$ & $0.002^{*}$ \\
\hline \multicolumn{10}{|l|}{ Parity } \\
\hline Parity 0 & & & & & & & & Ref. & \\
\hline Parity 1 & & & & & & & 0.33 & $0.15,0.50$ & $<0.001^{*}$ \\
\hline Parity $\geq 2$ & & & & & & & 0.32 & $0.13,0.52$ & $0.001^{*}$ \\
\hline \multicolumn{10}{|l|}{ Gestational age } \\
\hline Gestational age at birth (weeks) & & & & & & & 0.18 & $0.12,0.24$ & $<0.001^{*}$ \\
\hline$R^{2}$ per model & & 0.008 & & & 0.035 & & & 0.137 & \\
\hline
\end{tabular}

Ref., referent values; ART, antenatal antiretroviral treatment.

* Significant results $(P<0.05)$

$\dagger$ Multiple linear regression analyses.

Table 3. Hierarchical regression for the associations between maternal factors and neonatal fat mass index ( $n$ 171)

(Regression coefficients $(\beta)$ and $95 \%$ confidence intervals)

\begin{tabular}{|c|c|c|c|c|c|c|c|c|c|}
\hline \multirow[b]{3}{*}{ Independent variables } & \multicolumn{9}{|c|}{ Fat mass index $\left(\mathrm{kg} / \mathrm{m}^{3}\right)$} \\
\hline & \multicolumn{3}{|c|}{ Model 1} & \multicolumn{3}{|c|}{ Model 2} & \multicolumn{3}{|c|}{ Model 3} \\
\hline & $\beta$ & $95 \% \mathrm{Cl}$ & $P \dagger$ & $\beta$ & $95 \% \mathrm{Cl}$ & $P \dagger$ & $\beta$ & $95 \% \mathrm{Cl}$ & $P \dagger$ \\
\hline \multicolumn{10}{|l|}{ Neonate sex } \\
\hline Male & & Ref. & & & Ref. & & & Ref. & \\
\hline Female & 0.44 & $0.04,0.84$ & $0.033^{*}$ & 0.40 & $0.00,0.79$ & $0.049^{*}$ & 0.39 & $0.01,0.78$ & $0.044^{*}$ \\
\hline \multicolumn{10}{|l|}{ Maternal dietary pattern } \\
\hline Traditional pattern score & -0.09 & $-0.22,0.03$ & $0 \cdot 130$ & $-0 \cdot 10$ & $-0.22,0.02$ & 0.086 & $-0 \cdot 13$ & $-0.25,-0.02$ & $0.027^{*}$ \\
\hline \multicolumn{10}{|l|}{ HIV/treatment status } \\
\hline HIV-negative & & & & & Ref. & & & Ref. & \\
\hline HIV-positive (antenatal ART) & & & & 0.37 & $-0.10,0.84$ & 0.123 & 0.29 & $-0.17,0.75$ & 0.218 \\
\hline HIV-positive (pre-pregnancy ART) & & & & $1 \cdot 18$ & $0.48,1.87$ & $0.001^{\star}$ & $1 \cdot 11$ & $0.42,1.81$ & $0.002^{*}$ \\
\hline \multicolumn{10}{|l|}{ Gestational weight gain } \\
\hline Rate (kg/week) & & & & 0.44 & $-0.29,1.18$ & 0.238 & 0.46 & $-0.26,1.18$ & 0.213 \\
\hline \multicolumn{10}{|l|}{ BMI category } \\
\hline Normal weight & & & & & Ref. & & & Ref. & \\
\hline Overweight & & & & $0 \cdot 17$ & $-0.30,0.65$ & 0.469 & 0.20 & $-0.26,0.66$ & 0.390 \\
\hline Obese & & & & 0.37 & $-0.13,0.87$ & 0.147 & 0.24 & $-0.25,0.74$ & 0.335 \\
\hline \multicolumn{10}{|l|}{ Parity } \\
\hline Parity 0 & & & & & & & & Ref & \\
\hline Parity 1 & & & & & & & $0 \cdot 76$ & $0.27,1 \cdot 25$ & $0.002^{*}$ \\
\hline Parity $\geq 2$ & & & & & & & 0.64 & $0.11,1.17$ & $0.018^{*}$ \\
\hline \multicolumn{10}{|l|}{ Neonate age } \\
\hline Gestational age at birth (weeks) & & & & & & & 0.09 & $-0.08,0.26$ & 0.286 \\
\hline Age at examination (d) & & & & & & & 0.05 & $0.00,0.10$ & $0.035^{*}$ \\
\hline$R^{2}$ per model & & 0.043 & & & $0 \cdot 121$ & & & 0.194 & \\
\hline
\end{tabular}

Ref., referent values; ART, antenatal antiretroviral treatment.

* Significant results $(P<0.05)$.

$\dagger$ Multiple linear regression analyses. 
pattern score, HIV/treatment status, BMI, GWG, parity, gestational age at delivery and age at scan). HIV-positive (prepregnancy ART) $v$. HIV-negative status was associated with $1.18 \mathrm{~kg} / \mathrm{m}^{3}(P=0.001)$ higher neonatal FMI in M2 (adjusted for neonate sex, Traditional diet pattern score, HIV/treatment status, BMI, GWG). This association remained significant in the fully adjusted model (M3: $\left.1 \cdot 11 \mathrm{~kg} / \mathrm{m}^{3} ; P=0 \cdot 002\right)$. Approximately $19 \%$ of the variation in newborn FMI was explained in $\mathrm{M} 3$.

Although BMI and GWG were not associated with newborn FMI in any of the presented regression models, we confirmed an interaction between Traditional diet pattern adherence and maternal BMI on FMI ( $v$. normal weight: overweight $\times$ Traditional diet pattern, $P=0 \cdot 203$; obese $\times$ Traditional diet pattern, $P=0.024)$. Therefore, while dietary pattern adherence was associated with a significant reduction in FMI for infants born to normal-weight women $\left(-0.32 \mathrm{~kg} / \mathrm{m}^{3} ; P=0.005\right)$, these effects were not seen among newborns of overweight or obese women (data not shown)

\section{Discussion}

To our knowledge, this is the first study to explore the relationships between maternal nutritional status, dietary patterns and HIV/treatment exposure in African women and to examine their effects on neonatal adiposity within the first 2 weeks of life. We found that, although maternal obesity and GWG were associated with neonatal body composition, they predicted overall birth size rather than increased fat mass in particular. In contrast, adherence to a Traditional dietary pattern during pregnancy was associated with lower WLR and FMI, suggestive of a predominant effect on fat mass in the neonate. Finally, we showed that duration of ART exposure (pre-pregnancy $v$. antenatal initiation) in HIV-positive women was positively associated with newborn adiposity in this setting.

Our findings build on data previously reported on the association between Traditional dietary pattern adherence and reduced GWG (including odds of excessive weight gain) in this population $^{(10)}$. Here we show that not only is a dietary pattern high in vegetables, beans and legumes, traditional meats and whole grains associated with beneficial reductions in maternal adiposity during pregnancy but also in fetal fat deposition. Such effects have important implications for the long-term health trajectory of the infant, with previous studies showing a tendency towards tracking of adiposity through infancy, as well as of an increased risk of obesity and elevated metabolic risk profiles (including higher fasting TAG concentrations and insulin resistance) in later life ${ }^{(8,9)}$. In addition, while Catalano et $a l .{ }^{(9)}$ demonstrated a significant correlation between body fat percentage at birth and at childhood follow-up (mean age: 8.8 (sE 1.8) years), there was no correlation in total weights at these two time points. This highlights the importance of neonatal adiposity as a potential predictor for longer-term obesity and metabolic disease risk.

Although the associations between a traditional diet pattern and neonatal WLR and FMI are unique to our study, they are supported by Starling et al. who found that intake of a diet pattern with lower consumption of green vegetables and dairy and higher in refined grains was associated with higher birth weight, fat mass and body fat percentage in the USA ${ }^{(38)}$. However, conflicting results have also been shown, with typically healthier diet patterns (Mediterranean and Traditional) being associated with higher birth weight and reduced SGA risk, whereas more processed or Western dietary patterns have been associated with increased risk of SGA and lower weightfor-age $z$ score in some high-income settings ${ }^{(39-43)}$.

The relationship between maternal diet and birth size and adiposity is complex and the variation in findings across populations may reflect an influence of baseline nutritional status on these associations. For example, while energy-dense, processed diets have been shown to restrict fetal growth and increase SGA risk in certain populations, they may increase the risk of high birth weight and adiposity in others ${ }^{(42-44)}$. As both ends of the spectrum - that is being born too small or too large for gestational age - are associated with long-term disease risk $^{(45-48)}$, identifying patterns of intake that facilitate optimal fetal growth and limit excess fat deposition in increasingly urbanised African populations is critical. Given the high level of urbanisation in Africa - and particularly South Africa - to date, as well as the representativeness of the study population to that of an urban-poor community, our findings contribute substantially to understanding these contexts. Here we confirm that not only do the effects of maternal dietary patterns on GWG differ across BMI categories ${ }^{(10)}$ but the association between Traditional diet pattern adherence and neonatal adiposity is similarly modified by maternal BMI at baseline, with the effects seen predominantly in the normal-weight subgroup. This suggests that, in increasingly obesogenic populations, the beneficial effects of improved diet quality may be limited in women who exhibit excess adiposity and associated metabolic risk profiles before conception. Obesity induces a chronic inflammatory state that has been suggested as a key driver of insulin resistance and may intensify the naturally occurring insulinresistant profile during pregnancy ${ }^{(6,49,50)}$. Reduced insulin sensitivity in obese pregnant women - and the subsequent increase in availability of glucose and lipids - may potentially facilitate excess substrate transfer to the fetus and, thus, fat deposition irrespective of the current dietary pattern.

Although we found no differences in WLR or FMI between infants born to HIV-positive women with antenatal ART initiation compared with their HIV negative counterparts, we showed a significant increase in FMI for infants whose mothers were HIV-positive and initiated on ART before the current pregnancy. These associations were independent of baseline BMI, GWG and diet, and therefore suggest a strong treatment effect in this population. ART-associated metabolic complications have been widely documented in HIV-positive patients, with fat redistribution (reduced subcutaneous and increased central adiposity), impaired glucose tolerance and insulin resistance, as well as dyslipidaemia, being common side effects $^{(51,52)}$. Such changes may substantially affect the metabolic risk profile of women before conception, further exacerbating the pregnancy-associated insulin-resistant state and increasing the risk of gestational diabetes mellitus (GDM) and associated complications. These findings have important implications for HIV-positive pregnancies in South Africa, 
during which duration of ART exposure (increasing with each subsequent pregnancy) may elevate the risk profile for both mother and infant in an already high-risk population. Targeted monitoring and care strategies are therefore needed in order to minimise the adverse effects of treatment exposure on adiposity and associated metabolic risk in the newborn, with interventions designed to optimise nutritional status and diet quality before conception being potentially more vital in these women.

Although we showed significant effects of maternal nutritional status, diet and HIV/treatment exposure on birth size and/or neonatal adiposity, our final models explained only 14 and $19 \%$, respectively, of the variability in infant outcomes. While maternal adult size, adiposity and metabolic profile are predictive of infant outcomes, these characteristics are highly influenced by a mothers own growth and development, with studies showing strong intergenerational associations between maternal and offspring birth weight ${ }^{(53-56)}$. This suggests that the gestational environment of the mother - and the resulting consequences on maternal birth size and longer-term metabolic risk profile - may be an important factor in further explaining differences in neonatal size and adiposity in our study. In addition, there may be other behavioural factors that require exploration in these models, such as physical activity. Although there was no association found between physical activity and birth outcomes (including birth weight and ponderal index) in a previous study of this population ${ }^{(57)}$, any potential indirect effects on birth size - for example through reductions in GWG and/or risk of GDM -and possible associations with fetal fat deposition and neonatal adiposity should be explored ${ }^{(58-60)}$.

Other limitations of our study include the use of baseline BMI as a proxy for pre-pregnancy BMI and the variation in timing of maternal anthropometric measurements for assessing maternal GWG as previously described ${ }^{(10)}$. Although first trimester weight has been identified as an adequate proxy for prepregnancy weight - correctly classifying 91-95\% of women according to pre-pregnancy BMI - BMI-specific differences in weight gain during trimester one have been shown ${ }^{61,62)}$. Although the use of baseline weight may have resulted in a degree of misclassification in our study, any effects on study findings were likely to be negligible owing to the low overall amount of weight gained before 14 weeks, particularly in black women at higher $\mathrm{BMI}^{(62)}$. In addition, although the categorisation of pregnant women into three HIV/treatment status groups (i.e. HIV negative, HIV positive (antenatal ART) and HIV positive (pre-pregnancy ART)) allowed for effective comparison of overall treatment exposure in our sample, inclusion of additional measures of duration and/or adherence to ART would allow for more robust comparison of ART exposure on a continuous scale. While we present strong evidence for an effect of ART exposure on neonatal adiposity, the use of an objective measure such as viral load, an established proxy for ART adherence/effectiveness ${ }^{(63)}$, would be beneficial in further explaining the influence of treatment on fetal fat deposition and metabolic risk in future research. Given the focus of our study and the differential patterns of growth (both in fat mass and fatfree mass) between pre-term and term infants, we included only term neonates in our analyses ${ }^{(27)}$. Although this reduced the final sample size, it allowed for better interpretation of the effects of maternal factors (principally diet and HIV/treatment status) on newborn adiposity. However, future studies should explore these associations in pre-term infants in order to further elucidate the relationships with newborn size and adiposity according to gestational age. Last, neonatal body composition was measured using two techniques (Peapod and DXA) within the first 2 weeks of life, which may reduce comparability across the sample, particularly owing to the reduced sample size for these objective assessments. However, the comparability between techniques previously shown in this population allowed for correction of DXA to Peapod measurements and therefore equivalent fat mass estimates in the sub-sample ${ }^{(28)}$. Although the variation in day of neonatal body composition assessment must be considered - with physiological weight loss occurring during this period - any changes in body composition are predominantly due to reductions in body water and would therefore have little effect on the comparability of fat mass estimates between subjects ${ }^{(64)}$.

\section{Conclusions}

Our findings suggest that increased adherence to a Traditional diet pattern - high in whole grains, beans and legumes, vegetables and traditional meats and low in processed/convenience foods - may reduce neonatal adiposity in urban, black South Africans. However, early intervention to ensure a healthy BMI before pregnancy is needed in order to optimise the beneficial effects of diet quality on adiposity and associated metabolic risk for both mother and infant. Although ART initiation and adherence is critical for both maternal and infant health, the effects of treatment exposure on maternal metabolic risk and neonatal adiposity highlight the vulnerability of HIV-positive pregnant women and the importance of tailored care in this population. Targeted monitoring and management strategies are therefore necessary to limit treatment-associated effects on in utero fat deposition and to potentially reduce metabolic risk profiles and poor health trajectories of infants in both the short and longer term.

\section{Acknowledgements}

The support of the DST-NRF Centre of Excellence (CoE) in Human Development at the University of the Witwatersrand, Johannesburg, in the Republic of South Africa towards this research is hereby acknowledged. Opinions expressed and conclusions arrived at are those of the author and are not to be attributed to the CoE in Human Development.

In addition, we acknowledge funding from the UK MRC/DfID African Research Leader Scheme and the South African Medical Research Council. The aforementioned funders had no role in the design, analysis or writing of this article.

S. V. W., K. K. O. and S. A. N. contributed to conceptualisation and design of the work towards this paper; S. V. W., P. T. P. and S. A. N. facilitated data acquisition; S. V. W. analysed the data and drafted the manuscript; all authors contributed to interpretation of results and/or revision of the manuscript; all authors gave their approval of the final version for submission.

None of the authors has any conflicts of interest to declare. 


\section{Supplementary material}

For supplementary material/s referred to in this article, please visit https://doi.org/10.1017/S0007114518001708

\section{References}

1. Black RE, Victora CG, Walker SP, et al. (2013) Maternal and child undernutrition and overweight in low-income and middle-income countries. Lancet 382, 427-451.

2. Ruager-Martin R, Hyde MJ \& Modi N (2010) Maternal obesity and infant outcomes. Early Hum Dev 86, 715-722.

3. Wrottesley SV, Lamper C \& Pisa PT (2016) Review of the importance of nutrition during the first 1000 days: maternal nutritional status and its associations with fetal growth and birth, neonatal and infant outcomes among African women. J Dev Orig Health Dis 7, 144-162.

4. Poston L, Caleyachetty R, Cnattingius S, et al. (2016) Preconceptional and maternal obesity: epidemiology and health consequences. Lancet Diabetes Endocrinol 4, 1025-1036.

5. Hunt KJ, Alanis MC, Johnson ER, et al. (2013) Maternal prepregnancy weight and gestational weight gain and their association with birthweight with a focus on racial differences. Matern Child Health J 17, 85-94.

6. Catalano PM, Thomas A, Huston-Presley L, et al. (2003) Increased fetal adiposity: a very sensitive marker of abnormal in utero development. Am J Obstet Gynecol 189, 1698-1704.

7. Donnelly JM, Lindsay KL, Walsh JM, et al. (2015) Fetal metabolic influences of neonatal anthropometry and adiposity. BMC Pediatr 15, 175.

8. Ay L, Hokken-Koelega ACS, Mook-Kanamori DO, et al. (2008) Tracking and determinants of subcutaneous fat mass in early childhood: the Generation R Study. Int J Obes (Lond) 32 , 1050-1059.

9. Catalano PM, Farrell K, Thomas A, et al. (2009) Perinatal risk factors for childhood obesity and metabolic dysregulation. Am J Clin Nutr 90, 1303-1313.

10. Wrottesley SV, Pisa PT \& Norris SA (2017) The influence of maternal dietary patterns on body mass index and gestational weight gain in urban black South African women. Nutrients 9, 732 .

11. Popkin BM \& Gordon-Larsen $P$ (2004) The nutrition transition: worldwide obesity dynamics and their determinants. Int $J$ Obes Relat Metab Disord 28, Suppl. 3, S2-S9.

12. Popkin BM, Adair LS \& Ng SW (2012) Now and then: the global nutrition transition: the pandemic of obesity in developing countries. Nutr Rev 70, 3-21.

13. da Cunha J, Maselli LMF, Stern ACB, et al. (2015) Impact of antiretroviral therapy on lipid metabolism of human immunodeficiency virus-infected patients: old and new drugs. World J Virol 4, 56-77.

14. Koethe JR, Grome H, Jenkins CA, et al. (2016) The metabolic and cardiovascular consequences of obesity in persons with HIV on long-term antiretroviral therapy. AIDS Lond Engl 30, 83-91.

15. Maganga E, Smart LR, Kalluvya S, et al. (2015) Glucose metabolism disorders, HIV and antiretroviral therapy among Tanzanian adults. PLOS ONE 10, e0134410.

16. Li N, Sando MM, Spiegelman D, et al. (2016) Antiretroviral therapy in relation to birth outcomes among HIV-infected women: a cohort study. J Infect Dis 213, 1057-1064.

17. Kim RJ \& Rutstein RM (2010) Impact of antiretroviral therapy on growth, body composition and metabolism in pediatric HIV patients. Pediatr Drugs 12, 187-199.
18. Griffiths PL, Johnson W, Cameron $\mathrm{N}$, et al. (2013) In urban South Africa, 16 year old adolescents experience greater health equality than children. Econ Hum Biol 11, 502-514.

19. Kagura J, Adair LS, Pisa PT, et al. (2016) Association of socioeconomic status change between infancy and adolescence, and blood pressure, in South African young adults: Birth to Twenty Cohort. BMJ Open 6, e008805.

20. Institute of Medicine \& National Research Council (2009) Weight Gain During Pregnancy: Reexamining the Guidelines. Washington, DC: The National Academies Press. http://www. nap.edu/download.php?record_id=12584 (accessed June 2015).

21. Nel J \& Steyn N (2002) Report on South African Food Consumption Studies Undertaken Among Different Population Groups (1983-2000): Average Intakes of Foods Most Commonly Consumed. Pretoria: Department of Health.

22. Steyn NP, Senekal M, Norris SA, et al. (2006) How well do adolescents determine portion sizes of foods and beverages? Asia Pac J Clin Nutr 15, 35-42.

23. Dennis LK, Snetselaar LG, Nothwehr FK, et al. (2003) Developing a scoring method for evaluating dietary methodology in reviews of epidemiologic studies. J Am Diet Assoc 103, 483-487.

24. Zingoni C, Norris SA, Griffiths PL, et al. (2009) Studying a population undergoing nutrition transition: a practical case study of dietary assessment in urban South African adolescents. Ecol Food Nutr 48, 178-198.

25. Wrottesley SV, Micklesfield LK, Hamill MM, et al. (2014) Dietary intake and body composition in HIV-positive and -negative South African women. Public Health Nutr 17, 1603-1613.

26. Harris PA, Taylor R, Thielke R, et al. (2009) Research electronic data capture (REDCap) - A metadata-driven methodology and workflow process for providing translational research informatics support. J Biomed Inf 42, 377-381.

27. Villar J, Puglia FA, Fenton TR, et al. (2017) Body composition at birth and its relationship with neonatal anthropometric ratios: the newborn body composition study of the INTERGROWTH-21st project. Pediatr Res 82, 305-316.

28. Wrottesley SV, Pisa PT, Micklesfield LK, et al. (2016) A comparison of body composition estimates using dual-energy X-ray absorptiometry and air-displacement plethysmography in South African neonates. Eur J Clin Nutr 70, 1254-1258.

29. Hamill MM, Ward KA, Pettifor JM, et al. (2013) Bone mass, body composition and vitamin D status of ARV-naïve, urban, black South African women with HIV infection, stratified by CD4 count. Osteoporos Int 24, 2855-2861.

30. Urlando A, Dempster P \& Aitkens S (2003) A new air displacement plethysmograph for the measurement of body composition in infants. Pediatr Res 53, 486-492.

31. Yao M, Nommsen-Rivers L, Dewey K, et al. (2003) Preliminary evaluation of a new pediatric air displacement plethysmograph for body composition assessment in infants. Acta Diabetol 40, Suppl. 1, S55-S58.

32. Fomon SJ, Haschke F, Ziegler EE, et al. (1982) Body composition of reference children from birth to age 10 years. Am J Clin Nutr 35, 5 Suppl., 1169-1175.

33. Koo WW, Massom LR \& Walters J (1995) Validation of accuracy and precision of dual energy X-ray absorptiometry for infants. J Bone Miner Res 10, 1111-1115.

34. Crozier SR, Robinson SM, Borland SE, et al. (2006) Dietary patterns in the Southampton Women's Survey. Eur J Clin Nutr 60, 1391-1399.

35. Crozier SR, Inskip HM, Godfrey KM, et al. (2008) Dietary patterns in pregnant women: a comparison of food frequency questionnaires and four-day prospective diaries. Br J Nutr 99 , 869-875. 
36. Tielemans MJ, Erler NS, Leermakers ETM, et al. (2015) A priori and a posteriori dietary patterns during pregnancy and gestational weight gain: the Generation R study. Nutrients 7, 9383-9399.

37. Englund-Ögge L, Brantsæter AL, Sengpiel V, et al. (2014) Maternal dietary patterns and preterm delivery: results from large prospective cohort study. BMJ 348, g1446.

38. Starling AP, Sauder KA, Kaar JL, et al. (2017) Maternal dietary patterns during pregnancy are associated with newborn body composition. J Nutr 147, 1334-1339.

39. Northstone K, Ness AR, Emmett PM, et al. (2008) Adjusting for energy intake in dietary pattern investigations using principal components analysis. Eur J Clin Nutr 62, 931-938.

40. Timmermans S, Steegers-Theunissen RP, Vujkovic M, et al. (2012) The Mediterranean diet and fetal size parameters: the Generation R Study. Br J Nutr 108, 1399-1409.

41. Thompson JMD, Wall C, Becroft DMO, et al. (2010) Maternal dietary patterns in pregnancy and the association with smallfor-gestational-age infants. Br J Nutr 103, 1665-1673.

42. Knudsen VK, Orozova-Bekkevold IM, Mikkelsen TB, et al. (2008) Major dietary patterns in pregnancy and fetal growth. Eur J Clin Nutr 62, 463-470.

43. Colón-Ramos U, Racette SB, Ganiban J, et al. (2015) Association between dietary patterns during pregnancy and birth size measures in a diverse population in Southern US. Nutrients 7, 1318-1332.

44. Coelho N, de LP, Cunha DB, et al. (2015) Dietary patterns in pregnancy and birth weight. Rev Saúde Pública 49, 62.

45. Meas T, Deghmoun S, Armoogum P, et al. (2008) Consequences of being born small for gestational age on body composition: an 8-year follow-up study. J Clin Endocrinol Metab 93, 3804-3809.

46. Mericq V, Martinez-Aguayo A, Uauy R, et al. (2017) Long-term metabolic risk among children born premature or small for gestational age. Nat Rev Endocrinol 13, 50-62.

47. Harder T, Rodekamp E, Schellong K, et al. (2007) Birth weight and subsequent risk of type 2 diabetes: a meta-analysis. $\mathrm{Am} \mathrm{J}$ Epidemiol 165, 849-857.

48. Boney CM, Verma A, Tucker R, et al. (2005) Metabolic syndrome in childhood: association with birth weight, maternal obesity, and gestational diabetes mellitus. Pediatrics 115, e290-e296.

49. Esser N, Legrand-Poels S, Piette J, et al. (2014) Inflammation as a link between obesity, metabolic syndrome and type 2 diabetes. Diabetes Res Clin Pract 105, 141-150.

50. Shoelson SE, Herrero L \& Naaz A (2007) Obesity, inflammation, and insulin resistance. Gastroenterology 132, 2169-2180.
51. Grinspoon S \& Carr A (2005) Cardiovascular risk and body-fat abnormalities in HIV-infected adults. N Engl J Med 352, 48-62.

52. Stanley TL \& Grinspoon SK (2012) Body composition and metabolic changes in HIV-infected patients. J Infect Dis $\mathbf{2 0 5}$, Suppl. 3, S383-S390.

53. Kuzawa CW \& Eisenberg DTA (2012) Intergenerational predictors of birth weight in the Philippines: correlations with mother's and father's birth weight and test of maternal constraint. PLOS ONE 7, e40905.

54. Currie J \& Moretti E (2007) Biology as destiny? Short- and longrun determinants of intergenerational transmission of birth weight. J Labor Econ 25, 231-264.

55. Magnus P, Gjessing HK, Skrondal A, et al. (2001) Paternal contribution to birth weight. J Epidemiol Community Health 55, 873-877.

56. Simon DM, Vyas S, Prachand NG, et al. (2006) Relation of maternal low birth weight to infant growth retardation and prematurity. Matern Child Health J 10, 321-327.

57. Watson ED, Brage S, White T, et al. (2018) The influence of objectively measured physical activity during pregnancy on maternal and birth outcomes in urban black South African women. Matern Child Health J (epublication ahead of print version 7 March 2018).

58. Pearson JT, Watson ED, Lambert EV, et al. (2015) The role of physical activity during pregnancy in determining maternal and foetal outcomes. South Afr J Sports Med 27, 93-96.

59. Jiang H, Qian X, Li M, et al. (2012) Can physical activity reduce excessive gestational weight gain? Findings from a Chinese urban pregnant women cohort study. Int J Behav Nutr Phys Act 9, 12.

60. Clapp JF (2000) Exercise during pregnancy. A clinical update. Clin Sports Med 19, 273-286.

61. Krukowski RA, West DS, DiCarlo M, et al. (2016) Are early first trimester weights valid proxies for preconception weight? BMC Pregnancy Childbirth 16, 357.

62. Fontaine PL, Hellerstedt WL, Dayman CE, et al. (2012) Evaluating BMI-specific trimester weight gain recommendations: differences between black and white women. J Midwifery Womens Health 57, 327-335.

63. Myer L, Essajee S, Broyles LN, et al. (2017) Pregnant and breastfeeding women: a priority population for HIV viral load monitoring. PLOS Med 14, e1002375.

64. Toro-Ramos T, Paley C, Pi-Sunyer F, et al. (2015) Body composition during fetal development and infancy through the age of 5 years. Eur J Clin Nutr 69, 1279-1289. 